\title{
Lymphaticovenous Anastomosis for Lower Extremity Lymphedema: A Systematic Review
}

\author{
Antonio J. Forte ${ }^{1, \odot}$ Nawal Khan ${ }^{2}$ Maria T. Huayllani ${ }^{1}$ Daniel Boczar ${ }^{1}$ Humza Y. Saleem³ \\ Xiaona Lu ${ }^{4}$ Oscar J. Manrique ${ }^{5}$ Pedro Ciudad $^{6}$ Sarah A. McLaughlin ${ }^{3}$
}

${ }^{1}$ Division of Plastic Surgery, Mayo Clinic, Jacksonville, Florida, United States

2Ziauddin Hospital, Karachi, Pakistan

${ }^{3}$ Department of Surgery, Mayo Clinic, Jacksonville, Florida, United States

${ }^{4}$ Division of Plastic and Reconstructive Surgery, Yale School of

Medicine, New Haven, Connecticut, United States

${ }^{5}$ Division of Plastic Surgery, Mayo Clinic, Rochester, Minnesota, United States

${ }^{6}$ Department of Plastic, Reconstructive and Burn Surgery,

Arzobispo Loayza National Hospital, Lima, Peru
Address for correspondence Antonio J. Forte, MD, PhD, 4500 San Pablo Road, Jacksonville, Florida 32224, United States (e-mail: ajvforte@yahoo.com.br).

Indian J Plast Surg 2020;53:17-24

\begin{abstract}
Keywords

- lower extremity

- lymphaticovenous anastomosis

- lymphaticovenous bypass

- lymphedema

- lymphovenous anastomosis

- lymphovenous bypass

Background Lymphedema is an accumulation of protein-rich fluid in the interstitial spaces resulting from impairment in the lymphatic circulation that can impair quality of life and cause considerable morbidity. Lower extremity lymphedema (LEL) has an overall incidence rate of $20 \%$. Conservative therapies are the first step in treatment of LEL; however, they do not provide a cure because they fail to address the underlying physiologic dysfunction of the lymphatic system. Among several surgical alternatives, lymphaticovenous anastomosis (LVA) has gained popularity due to its improved outcomes and less invasive approach. This study aims to review the published literature on LVA for LEL treatment and to analyze the surgical outcomes.

Methods PubMed database was used to perform a comprehensive literature review of all articles describing LVA for treatment of LEL from Novemeber 1985 to June 2019. Search terms included "lymphovenous" OR "lymphaticovenous" AND "bypass" OR "anastomosis" OR "shunt” AND "lower extremity lymphedema."

Results A total of 95 articles were identified in the initial query, out of which 58 individual articles were deemed eligible. The studies included in this review describe notable variations in surgical techniques, number of anastomoses, and supplementary interventions. All, except one study, reported positive outcomes based on limb circumference and volume changes or subjective clinical improvement. The largest reduction rate in limb circumference and volume was $63.8 \%$.

Conclusion LVA demonstrated a considerable reduction in limb volume and improvement in subjective findings of lymphedema in the majority of patients. The maintained effectiveness of this treatment modality in long-term follow-up suggests great efficacy of LVA in LEL treatment.
\end{abstract}

DOI https://doi.org/

10.1055/s-0040-1709372 ISSN 0970-0358.
(C)2020 Association of Plastic Surgeons of India
License terms

()(1) $\Theta \circledast$ 


\section{Introduction}

Impairment in lymphatic circulation leads to the accumulation of protein-rich fluid in the interstitial spaces, giving rise to a chronic and debilitating condition known as lymphedema that is characterized by edema, inflammation, and cellulitis. Individuals suffering from lymphedema experience pain, fatigue, numbness, swelling, depression, limitation of daily activities, social impairment, and difficulty wearing normal clothing. ${ }^{1,2}$ These functional and cosmetic disabilities greatly affect their quality of life. Eventually, irreversible changes ensue, including fibrosis and proliferation of adipose tissue. ${ }^{2}$ In rare cases, continual angiogenic stimulus resulting from chronic lymphedema can manifest in the form of malignancies, such as lymphangiosarcoma (Stewart-Treves syndrome), Kaposi's sarcoma, and lymphoma. ${ }^{3}$

Primary lymphedema is characterized by a disruption in normal lymphatic fluid transport due to agenesis or dysgenesis of any component of the lymphatic network..$^{4-8}$ Occasionally, a lymphatic thrombus may be the cause. ${ }^{9}$ Secondary lymphedema is caused by mechanical obstruction of lymphatic drainage due to trauma, infection, radiation, or surgical disruption.,10-12

Lower extremity lymphedema (LEL) has an overall incidence of $20 \%{ }^{13}$ It is reported to occur in up to $21 \%$ of prostate, $16 \%$ of bladder, $4 \%$ of penile, $11.1 \%$ of ovarian, ${ }^{14} 12.6 \%$ to $27.0 \%$ of cervical, ${ }^{15} 13 \%$ of endometrial, ${ }_{16}^{16}$ and 16.7 to $30.0 \%$ of vulvar cancers after radical surgery. ${ }^{17-19}$ Several risk factors for LEL have been identified, including older age, higher body mass index, radiation therapy, cellulitis and wound infection, and the number of removed lymph nodes. ${ }^{18,20}$

Conservative therapies do not address the underlying dysfunction of the lymphatic drainage system and, hence, are ineffective in halting the progression of the disease. Surgical techniques, reconstructive or physiologic, target the functional repair of this intrinsic problem. Among these is a supermicrosurgical procedure known as lymphaticovenous anastomosis (LVA) or lymphovenous bypass that has gained wide spread acceptance worldwide due to its improved outcomes and less invasive approach. Anastomosing lymphatic channels to the adjacent venules redirects the lymphatic flow to the venous circulation, thereby draining the excess fluid trapped in the lymphedematous district. The lymphatic vessel and venule are anastomosed in an intima-to-intima coaptation fashion, leading to a lower anastomotic-site thrombosis rate. ${ }^{21}$ The outcome of this technique is influenced by the quality of the lymphatic vessels bypassed and the number of LVAs made. Functional smooth muscle cells lining the lymphatic channels are required to generate an effective pressure gradient to force the congested lymph into the venous system.22

This study aims to review the published literature on LVA in the treatment of LEL and to analyze the surgical outcomes. To our knowledge, this is the first review that includes all studies to date describing LVA surgery and its effect on primary and secondary lymphedema of lower extremities.

\section{Methods}

PubMed was queried for articles reporting LVA surgery in LEL treatment and their postsurgical outcomes using the

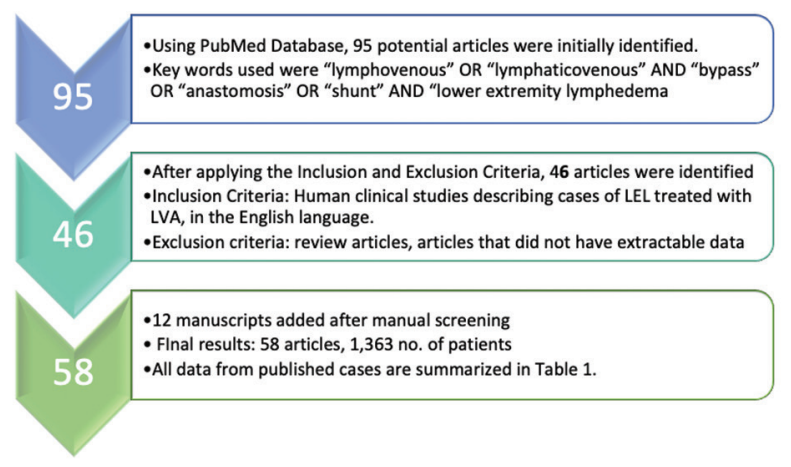

Fig. 1 Flowchart highlighting search strategy and article identification.

search terms, "lymphovenous" OR "lymphaticovenous" AND "bypass" OR "anastomosis" OR "shunt" AND "lower extremity lymphedema." Only human clinical studies in the English language were eligible for inclusion. Manuscripts mentioning both upper and lower limb lymphedema were included as long as their results included extractable data regarding outcomes of LVA for LEL treatment. Literature review articles were excluded. The publications were further screened manually and reviewed to identify additional reports for surgical treatment of LEL through LVA. The second author (N.K.) independently reviewed and extracted data from the papers according to the predetermined criteria. Demographic data, lymphedema duration, surgical technique, follow-up time, and outcomes were extracted from the selected articles.

\section{Results}

We found a total of 95 articles in our first search. From these, only 58 studies met inclusion criteria (-Fig. 1). The manuscripts included were published from November 1985 to July 2019 and reported data for a total of 1,363 patients with LEL who had undergone LVA - Supplementary Table S1, (available online only). The number of patients in each study ranged from $1^{23-27}$ to $216,{ }^{28}$ with a female predominance in all. The mean age at presentation ranged from $6^{29}$ to 94 years, ${ }^{30}$ and the mean duration of LEL ranged from 22 days $^{31}$ to 585 months. ${ }^{1}$ Patients included in the studies more commonly had secondary lymphedema.

Campisi's lymphedema grading system $^{32}$ was used in 12 studies, while the Yamamoto classification system ${ }^{33}$ based on lymphatic dermal backflow patterns seen on indocyanine green lymphography (ICG-L) was used in 17 studies. Mihara et al, ${ }^{34}$ Akita et $\mathrm{al}^{35}$ and Maegawa et al ${ }^{36}$ used the grading system based on lymphoscintigraphy findings, described by Maegawa et $\mathrm{al}^{37}$ in one of their earlier reports. Ito and collegues ${ }^{38}$ used Cheng's classification system ${ }^{39}$ and Olszewski ${ }^{28}$ used a grading system based on evaluation of the level of edema embracing the limb from foot to groin and the advancement of skin keratosis and fibrosis. However, the most widely used classification in 20 studies was the one presented by the International Society of Lymphology. ${ }^{40}$

The preoperative and operative detailsalong with the outcomes are summarized in -Supplementary Table S2 (available online only). Lower limb circumference and volume change were the most frequently assessed parameters, followed by subjective 
improvement. Mihara et $\mathrm{al}^{30}$ and Gloviczki et $\mathrm{al}^{41}$ used lymphoscintigraphy, while Chen and colleagues used ICG-L $\mathrm{L}^{42}$ to assess postoperative efficacy of LVA. Akita et al ${ }^{43}$ used computed tomography to calculate thickness of the subcutaneous fat layer on the lateral edge of the rectus femoris muscle at the level of the lesser trochanter of the femur and subsequently evaluated postoperative improvement of lymphedema.

ICG-L was the most commonly used investigation to assess the severity of lymphedema and preoperatively outline the location of lymphatic vessels. End-to-end (E-E) and sideto-end (S-E) anastomosis were the most frequently employed surgical techniques. A modified version of the E-E LVA, the sleeve-in technique, in which a lymphatic vessel is implanted into the lumen of a vein, was used by Yamamoto and Sugihara, ${ }^{44}$ Olszewski, ${ }^{28}$ and Chung et al. ${ }^{45}$

Yamamoto et $\mathrm{al}^{46}$ introduced the $\lambda$-shaped anastomosis with intravascular stent (IVaS) method for cases in which only one lymphatic channel and one venule could be found. They suggested identifying vessels appropriate for anastomosis and transecting them. After that, an IVaS, a piece of nylon monofilament suture, is placed in the vessels to keep the lumen open and assist safe anastomosis. An end-to-side (E-S) LVA followed by an E-E LVA is performed, creating a $\lambda$-shaped anastomosis. ${ }^{46}$ Furthermore, when only one venule and two lymphatic channels were found, they reported the use of sequential anastomosis, consisting of S-S and S-E LVA. Four directions of lymphatic flow can be diverted from two lymphatic vessels into one venule via this arrangement. ${ }^{47}$ They also described the use of ladder-shaped anastomosis for cases when only one vein and three lymphatic vessels were identified intraoperatively. ${ }^{48}$ In this technique, the two lymphatic vessels next to the vein are anastomosed to the vein in a S-S fashion, and the other lymphatic vessel is anastomosed to the nearby lymphatic vessel in a S-S fashion.

Ayestaray and Bekara ${ }^{49}$ developed a configuration based on two E-S LVA, or $\pi$-shaped LVA. They called it a venous flow-sparing technique that resulted in an average circumferential differential reduction rate of $19.4 \%$ (range, $6.1-24.6 \%$ ). ${ }^{49}$ In an attempt to solve the problem of mismatch between the number of lymphatic vessels and venules and their calibers, Chen et $a^{50}$ presented the octopus LVA. This technique allows multiple lymphatic vessels to be bypassed using a single vein. An out-to-in transluminal suture is placed through a vein with a large diameter, followed by an in-to-out transluminal suture, which intussuscepts all the lymphatic vessels into the vein, and forms an LVA complex that resembles an octopus. ${ }^{50}$

Per case $1^{29,30,36,51-53}$ to $18^{46}$ anastomoses were performed, and it took an average of 3 to 4 hours ${ }^{29,35,44-46,54-58}$ for surgeons to perform the entire procedure. Follow-up time ranged from $1^{59,60}$ to 87 months. ${ }^{61}$ Complex decongestive physical therapy was advised to be continued postoperatively in most cases. All studies reported positive outcomes based on improvement in the limb circumference and volume, lymphatic function, or clinical symptoms. Ito et al, ${ }^{38}$ Koshima et al, ${ }^{62}$ and Lee et $\mathrm{a}^{63}$ have reported the largest reduction rates at 63.8 , 55.6 , and $51.1 \%$, respectively.

Regarding outcomes, Gloviczki and collegues ${ }^{41}$ reported improvement in only two of eight patients, while four patients reported worsening of lymphedema. Mihara et $\mathrm{al}^{34}$ compared LVA outcomes in the following two groups of patients: those in whom noncontact vein visualizing system was used to detect subcutaneous veins (control group) versus those in whom venous echography was used (echo group). Lymphedema exacerbation was reported in $23.8 \%$ of the patients from the control group, while only $5.9 \%$ from the echo group experienced lymphedema deterioration. ${ }^{34}$ In patients with lymphedema onset before 11 years of age, Hara et $\mathrm{a}^{58}$ reported aggravation of their condition. Positive outcomes were seen more often in those with onset after 11 years of age. ${ }^{58}$

The largest number of patients included in a study was reported by Olszewski. ${ }^{28}$ Over the course of 45 years, the author performed LVAs on 216 patients, with an average postoperative follow-up of 60 months. Major improvement was observed in patients with early stages of lymphedema, regardless of the etiology. The lymphovenous shunt operation was ineffective in patients with stage-II idiopathic and stage-III postinflammatory types of lymphedema. Hence, Olszewski suggested performing the surgery at an early stage (I and II) of postinflammatory, postsurgical, or hyperplastic types, with at least one thigh lymphatic and inguinal or iliac lymph node visible on stress lymphoscintigraphy.

Similarly, Demirtas et al ${ }^{60}$ reported a greater decrease in limb volume in patients with a lower volume of edema. However, Mihara et al ${ }^{30}$ described contradicting results, with significant volume reductions in advanced stages of lymphedema. They recorded a volume decline in $73.9 \%$ of patients with stage III compared with $56.7 \%$ with stage I.

Thirteen studies assessed the relationship between lymphedema and episodes of cellulitis or lymphangitis. ${ }^{1,24,27,29,30,34,38,56,60,61,63-65}$ All, except one chart review by Lee et $\mathrm{al}^{63}$ reported significant reductions in infection incidence.

\section{Discussion}

The possibility of creating an anastomosis between lymphatic vessels and veins to diverge the lymphatic fluid into the venous system was mentioned for the first time in $1962 .{ }^{66}$ Subsequently, several others attempted to perform LVA in canine models. ${ }^{67-69}$ However, the field of lymphatic microsurgery did not experience a major breakthrough until O'Brien et $\mathrm{al}^{70}$ presented a clinical report establishing the clinical effectiveness of this method in human patients. Lymphatic vessels with a diameter of 0.5 to $1.0 \mathrm{~mm}$ and veins with a diameter of 2.0 to $3.0 \mathrm{~mm}$ were required for anastomosis under general anesthesia. ${ }^{70}$ Thereafter, the introduction of supermicrosurgery, which enabled anastomosis of vessels with a caliber of 0.3 to $0.8 \mathrm{~mm}$, increased the reliability of LVA. ${ }^{62.71}$ LVA using lymphatics with diameter between 0.5 to $0.7 \mathrm{~mm}$ and subdermal veins between 0.7 to $1.0 \mathrm{~mm}$ of diameter demonstrated positive outcomes with reduction rates from 30 to $78 \%$ and reduction of episodes of cellulitis from a mean of 1.6 to $0.2 .^{38}$ Currently, the operation can be performed under local anesthesia in the dermal layer of the skin via a 2- to 3-cm skin incision, making it safe for high-risk patients, including the elderly and those with 
cardiopulmonary disease or terminal cancer. ${ }^{62}$ Mihara et al ${ }^{72}$ was able to report favorable outcomes of LVA performed through a 2-mm skin incision under guidance of the AccuVein system (AccuVein Inc.) and ICG-L in patients with early and latent stage lymphedema. This was possible because of the absence of subcutaneous tissue fibrosis in these patients.

LVA is indicated in cases where there has been insufficient lymphedema reduction by complex physical therapy (less than $50 \%$, worsening limb function, recurrent episodes of cellulitis, and intractable pain. In addition, patients not satisfied with the result obtained by conservative methods can be given the option to proceed with a surgical alternative. ${ }^{73} \mathrm{LVA}$ can also be performed as prophylactic treatment in patients with subclinical lymphedema. ${ }^{72,74}$

Many surgeons agree that it is desirable to perform microlymphatic surgery at an early stage of edema because lymphatics get permanently damaged from increased pressure and recurrent infections, and preserved smooth muscle function is required to effectively pump the lymphatic fluid into the venous system. ${ }^{32,35,38,53,60,61,65,71,75-77}$ Thus, advanced disease, where lymphatic vessels are usually sclerotic, is a relative contraindication.

Other procedures used to treat lower limb lymphedema include vascularized lymph node transfer (VLNT), as well as VLNT in combination with lipoaspiration. ${ }^{78}$ Although, VLNT is also preferred in early stages of the disease as LVA, LVA is better suited in patients with available lymphatic vessels. ${ }^{78-80}$ In addition, LVA has the advantage of not having any morbidity risk associated with the donor site as in VLNT. ${ }^{79}$ On the other hand, combined procedures have shown better outcomes in more advanced stages of lymphedema. ${ }^{78}$

Previously, it was believed that primary lymphedema was also a relative contraindication for LVA due to the hypoplastic nature of lymphatic vessels. ${ }^{75}$ However, encouraging results have been reported for select patients with primary lymphedema in the recent years. ${ }^{58,81}$ Congenital lymph node fibrosclerosis and the lower number of functional lymphatic collectors can account for the decreased volume reduction observed in primary lymphedema as compared with secondary. ${ }^{32,81}$ Since extensive lymphatic abnormalities are associated with early onset of disease, LVA is advised in cases of primary lymphedema with no dermal backflow and disease onset after 11 years of age..$^{58}$

Regarding the surgical technique, subdermal venules with intact valves are recommended for anastomosis due to their small diameter and lower intravenous pressures. The smaller diameter allows caliber-matching, and lower intravenous pressure with a functioning valve reduces the chance of thrombosis due to minimal backflow. ${ }^{82}$

Constant lymphatic vessels are easier to locate at the dorsal foot, ankle, and groin region for the lower extremity. ${ }^{29}$ However, Seki et al ${ }^{83}$ described a method to locate easily between three to five superficial large lymphatic vessels when a $2.5-\mathrm{cm}$ transverse incision is made in the intersection of a transverse line at the superior edge of the patella and a longitudinal line along the medial axis of the distal thigh. Preoperative identification of functional lymphatic vessels and veins can substantially shorten operative time and improve surgical outcomes. ${ }^{84}$ For this purpose, ICG-L and lymphoscintigraphy can be used to locate patent lymphatic vessels and assess the severity of lymphedema before surgery. ${ }^{55,56,72,85-87}$ Although, our systematic review observed different classifications used to assess severity of lymphedema, the most widely used classification was the one stated by International Society of Lymphology. Ultrasound is a noninvasive tool that can also facilitate lymphatic channel visualization in regions masked by dermal backflow pattern or in patients allergic to iodine..$^{55,57}$ It has a sensitivity and specificity of 88.2 and $92.7 \%$, respectively. ${ }^{57}$ Postoperative patency and efficacy of the anastomosis can also be determined via lymphoscintigraphy ${ }^{41}$ and ICG-L. ${ }^{42}$ Lee et $\mathrm{al}^{63}$ published a chart review in which they suggested that once LVA is conducted properly and becomes functional in the early postoperative period, it might remain effective for up to 4 years following surgery.

Performing as many LVAs as possible is desirable, given that treatment efficacy was found to be proportional to the number of anastomoses created. ${ }^{38,46,65,82,86}$ However, there is still a topic open for investigation regarding the number of anastomoses that result in significant reductions. $\mathrm{Chen}^{88}$ in his operative technique that routinely they perform between 7 to 12 per limb. Koshima et $\mathrm{a}^{53}$ believe that a large number of LVAs is not required, and only two to three anastomoses are enough to provide a satisfactory volume reduction. Seki and colleagues, on the other hand, commented that one functional LVA can be sufficient. ${ }^{83}$

The basic types of LVA include E-E, E-S, S-E, and S-S. ${ }^{46,47,52,89-91}$ Controversy still exists regarding the type of anastomosis that should be performed but each type has its own advantages and disadvantages. E-E LVA seems to be inferior to E-S and S-S LVA as it drains only distal lymph in LEL and requires a higher number of anastomoses in more proximal sites. ${ }^{92}$ Moreover, lymphedema is characterized to have a retrograde and antegrade lymph flow, therefore, LVA should try to bypass both directions of lymph flows. ${ }^{92}$ S-E LVA is the most technically challenging and recommended, and is efficient to divert bidirectional lymph flow via one anastomosis. ${ }^{38}$ Similarly, S-S anastomosis can divert both antegrade and retrograde lymph flows, but it takes a longer time compared with S-E anastomosis and eventually results in venous backflow, which may cause anastomotic site thrombosis. However unlike S-E LVA, S-S LVA does not require supermicrosurgical technique when the diameter of the lymphatic vessel is smaller than $0.5 \mathrm{~mm}$, can be performed when the vein is much larger than the lymphatic vessel, and can use a vein distal to the anastomotic site. ,7,46,47,86,90 $^{37}$

Due to the small caliber of microvessels and the transparent nature of lymphatic vessels, the procedure of LVA is technically challenging. Yamamoto et $\mathrm{al}^{90}$ proposed the method of temporary lymphatic expansion during S-E anastomosis, whereby the lymphatic vessels are clamped and manually massaged. Although temporary, this maneuver can dilate the vessels by approximately $0.12 \mathrm{~mm}$, making the creation of a lateral window much easier. Another way to facilitate the anastomosis is by inserting an IVaS, a piece of nylon suture that allows identification of the lumen of lymph channels and stabilizes vessels while guiding the needle. ${ }^{46,91,93}$ Building on 
this approach, Yoshida et al ${ }^{54}$ illustrated the mechanical dilatation technique, in which a larger nylon monofilament was inserted after the IVaS to dilate the vessel lumen. According to their data, even a vessel with a diameter of less than $0.1 \mathrm{~mm}$ can be dilated to more than $0.2 \mathrm{~mm}$ using this method, with $100 \%$ immediate patency rate..$^{54}$ These techniques can aid in the creation of safe and secure LVAs in a shorter amount of time, thus increasing the number of successful LVAs that can be performed.

Supportive use of complex decongestive physical therapy should not be ignored after surgery. ${ }^{75,81}$ It consists only of compression therapy with the help of bandaging and elastic stockings but also of manual lymph drainage and massage, therapeutic exercise, and careful skin care. ${ }^{29}$ Superior outcomes have been observed in patients who underwent a combination of LVA and compression bandaging compared with those who underwent surgery alone. ${ }^{43}$ Compression therapy should be initiated one month postsurgery and patients should be follow up each 3 months postoperatively to assess volume reduction until maintenance of limb size is achieved with daytime therapy for 3 consecutive months. ${ }^{94}$

Our systematic review found that the largest reduction rates achieved after LVA for LEL ranged between 51.1 and 63.8\%, with better results presented in early stages of lymphedema, and that almost all studies reported a decrease in episodes of infection. Patients' satisfaction after LVA has been also evaluated. Chung et a ${ }^{45}$ found moderate scores of satisfaccion after treatment. Moreover, improvement in the quality of life has been demonstrated after LVA during the first postoperative months. ${ }^{1,42}$ Although, most of the patients who underwent LVA for LEL have been reported to have positive surgical outcomes, limb size reduction can be variable. This may be related with the severity of the lymphedema and the number and type of anastomoses performed along with the risk factors associated with lymphedema presented in the patients included in the studies. Several studies have reported inferior outcomes for patients who have undergone LVA for LEL compared with upper extremity lymphedema. ${ }^{38}$ The effects of ambulation, the large size, the dependent position, and the higher venous pressure of the lower extremities may account for this finding. ${ }^{29}$ When a volumetric decrease of the edema was not achieved, it constitutes a failure. In those cases, options such as VLNT, lipoaspiration or combined procedures could be considered.

\section{Limitations}

As with all systematic reviews, this study has several limitations. Due to the general lack of large volume studies addressing LVA in the lower extremity, we were restricted to a limited range of reported data, and hence, a thorough statistical analysis was not possible. Considerable heterogeneity exists among the reported outcomes in each study, and therefore, there is a potential for bias in interpreting data, as it is possible that not all studies captured reliable comorbidity data or outcomes over a long-period of time. A risk of selective reporting bias can also be encountered when documentation of subjective patient symptoms is involved. Larger, randomized, multicenter studies are warranted to validate the results found from this systematic review of the literature.

\section{Conclusion}

The results of this systematic review of the literature on LVA for LEL demonstrated considerable improvements in objective and subjective findings in the majority of patients. More importantly, the effectiveness of this treatment modality was maintained in the long-term follow-up, suggesting great efficacy of LVA in cases of LEL.

\section{Funding}

This study was supported in part by the Mayo Clinic Center for Individualized Medicine and the Plastic Surgery Foundation.

\section{Conflict of Interest}

A.J.F. reports others and this study was supported in part by the Mayo Clinic Robert D. and Patricia E. Kern Center for the Science of Health Care Delivery, and by the Mayo Clinic Center for Individualized Medicine. D.B. reports others and This study was supported in part by the Mayo Clinic Robert D. and Patricia E. Kern Center for the Science of Health Care Delivery, and by the Mayo Clinic Center for Individualized Medicine. H.Y.S. reports others and this study was supported in part by the Mayo Clinic Robert D. and Patricia E. Kern Center for the Science of Health Care Delivery, and by the Mayo Clinic Center for Individualized Medicine. M.T.H. reports others and this study was supported in part by the Mayo Clinic Robert D. and Patricia E. Kern Center for the Science of Health Care Delivery, and by the Mayo Clinic Center for Individualized Medicine. N.K. reports others and this study was supported in part by the Mayo Clinic Robert D. and Patricia E. Kern Center for the Science of Health Care Delivery, and by the Mayo Clinic Center for Individualized Medicine. O.J.M. reports others and this study was supported in part by the Mayo Clinic Robert D. and Patricia E. Kern Center for the Science of Health Care Delivery, and by the Mayo Clinic Center for Individualized Medicine. P.C. reports others and this study was supported in part by the Mayo Clinic Robert D. and Patricia E. Kern Center for the Science of Health Care Delivery, and by the Mayo Clinic Center for Individualized Medicine. S.A.McL. reports others and this study was supported in part by the Mayo Clinic Robert D. and Patricia E. Kern Center for the Science of Health Care Delivery, and by the Mayo Clinic Center for Individualized Medicine. X.L. reports others and This study was supported in part by the Mayo Clinic Robert D. and Patricia E. Kern Center for the Science of Health Care Delivery, and by the Mayo Clinic Center for Individualized Medicine.

\section{References}

1 Salgarello M, Mangialardi ML, Pino V, Gentileschi S, Visconti G. A prospective evaluation of health-related quality of life following lymphaticovenular anastomosis for upper and lower extremities lymphedema. J Reconstr Microsurg 2018;34(9):701-707 
2 Brorson H, Ohlin K, Olsson G, Nilsson M. Adipose tissue dominates chronic arm lymphedema following breast cancer: an analysis using volume rendered CT images. Lymphat Res Biol 2006;4(4):199-210

3 Ruocco V, Schwartz RA, Ruocco E. Lymphedema: an immunologically vulnerable site for development of neoplasms. J Am Acad Dermatol 2002;47(1):124-127

4 Becker C, Arrive L, Saaristo A, et al. Surgical treatment of congenital lymphedema. Clin Plast Surg 2012;39(4):377-384

5 Liu X, Pasula S, Song H, et al. Temporal and spatial regulation of epsin abundance and VEGFR3 signaling are required for lymphatic valve formation and function. Sci Signal 2014; 7(347):ra97

6 Rustgi AK, Rotolo FS, Peete WP, Vollmer RT, Meyers WC. Successful management of late-onset primary lymphatic hypoplasia. Surgery 1985;97(6):714-720

7 Hara H, Mihara M, Okuda I, et al. Presence of thoracic duct abnormalities in patients with primary lymphoedema of the extremities. J Plast Reconstr Aesthet Surg 2012;65(11):e305-e310

8 Hara H, Koshima I, Okuda I, Narushima M, Mihara M, Todokoro T. Assessment of configuration of thoracic duct using magnetic resonance thoracic ductography in idiopathic lymphedema. Ann Plast Surg 2012;68(3):300-302

9 Hara H, Mihara M, Seki Y, Koshima I. Lymphoedema caused by idiopathic lymphatic thrombus. J Plast Reconstr Aesthet Surg 2013;66(12):1780-1783

10 Garfein ES, Borud LJ, Warren AG, Slavin SA. Learning from a lymphedema clinic: an algorithm for the management of localized swelling. Plast Reconstr Surg 2008;121(2):521-528

11 Witte CL, Witte MH. Disorders of lymph flow. Acad Radiol 1995;2(4):324-334

12 Olszewski W. On the pathomechanism of development of postsurgical lymphedema. Lymphology 1973;6(1):35-51

13 Forte AJ, Huayllani MT, Boczar D, Ciudad P, McLaughlin SA. Lipoaspiration for the treatment of lower limb lymphedema: a comprehensive systematic review. Cureus 2019;11(10):e5913

14 Ki EY, Park JS, Lee KH, Hur SY. Incidence and risk factors of lower extremity lymphedema after gynecologic surgery in ovarian cancer. Int J Gynecol Cancer 2016;26(7):1327-1332

$15 \mathrm{Kim} \mathrm{JH}$, Choi JH, Ki EY, et al. Incidence and risk factors of lowerextremity lymphedema after radical surgery with or without adjuvant radiotherapy in patients with FIGO stage I to stage IIA cervical cancer. Int J Gynecol Cancer 2012;22(4):686-691

16 Beesley VL, Rowlands IJ, Hayes SC, et al; Australian National Endometrial Cancer Study Group. Incidence, risk factors and estimates of a woman's risk of developing secondary lower limb lymphedema and lymphedema-specific supportive care needs in women treated for endometrial cancer. Gynecol Oncol 2015;136(1):87-93

17 Cormier JN, Askew RL, Mungovan KS, Xing Y, Ross MI, Armer JM. Lymphedema beyond breast cancer: a systematic review and meta-analysis of cancer-related secondary lymphedema. Cancer 2010;116(22):5138-5149

18 Huang J, Yu N, Wang X, Long X. Incidence of lower limb lymphedema after vulvar cancer: A systematic review and meta-analysis. Medicine (Baltimore) 2017;96(46):e8722

19 Forte AJ, Huayllani MT, Boczar D, Cinotto G, McLaughlin SA. Ultrasound elastography use in lower extremity lymphedema: a systematic review of the literature. Cureus 2019;11(9):e5578

20 Hareyama H, Hada K, Goto K, et al. Prevalence, classification, and risk factors for postoperative lower extremity lymphedema in women with gynecologic malignancies: a retrospective study. Int J Gynecol Cancer 2015;25(4):751-757

21 Yamamoto T, Yamamoto N, Fuse Y, Narushima M, Koshima I. Optimal sites for supermicrosurgical lymphaticovenular anastomosis: an analysis of lymphatic vessel detection rates on 840 surgical fields in lower extremity lymphedema patients. Plast Reconstr Surg 2018;142(6):924e-930e
22 Visconti G, Salgarello M, Hayashi A. The recipient venule in supermicrosurgical lymphaticovenular anastomosis: flow dynamic classification and correlation with surgical outcomes. J Reconstr Microsurg 2018;34(8):581-589

23 Scaglioni MF, Uyulmaz S. Lymphovenous anastomosis and debulking procedure for treatment of combined severe lower extremity and genital lymphedema: A case report. Microsurgery 2018;38(8):907-911

24 Mihara M, Hara $\mathrm{H}$, Tsubaki $\mathrm{H}$, et al. Combined conservative treatment and lymphatic venous anastomosis for severe lower limb lymphedema with recurrent cellulitis. Ann Vasc Surg 2015;29(6):1318.e11-1318.e15

25 Mihara M, Zhou HP, Hara H, Tange S, Haragi M. Case report: a new hybrid surgical approach for treating mosaic pattern secondary lymphedema in the lower extremities. Ann Vasc Surg 2014;28(7):1798.e1-1798.e6

26 Mihara M, Murai N, Hayashi Y, et al. Popliteal lymphatico-venous anastomosis for lower limb lymphedema to allow patients to adopt the traditional Japanese "seiza" sitting position. J Plast Reconstr Aesthet Surg 2011;64(11):1544-1546

27 Suehiro K, Morikage N, Murakami M, Yamashita O, Hamano K. Primary lymphedema complicated by weeping chylous vesicles in the leg and scrotum: report of a case. Surg Today 2012;42(11):1100-1103

28 Olszewski WL. Lymphovenous microsurgical shunts in treatment of lymphedema of lower limbs: a 45-year experience of one surgeon/one center. Eur J Vasc Endovasc Surg 2013;45(3): 282-290

29 Pereira N, Lee YH, Suh Y, et al. Cumulative experience in lymphovenous anastomosis for lymphedema treatment: the learning curve effect on the overall outcome. J Reconstr Microsurg 2018;34(9):735-741

30 Mihara M, Hara H, Tange S, et al. Multisite lymphaticovenular bypass using supermicrosurgery technique for lymphedema management in lower lymphedema cases. Plast Reconstr Surg 2016;138(1):262-272

31 Auba C, Marre D, Rodríguez-Losada G, Hontanilla B. Lymphaticovenular anastomoses for lymphedema treatment: 18 months postoperative outcomes. Microsurgery 2012;32(4): 261-268

32 Campisi C, Davini D, Bellini C, et al. Lymphatic microsurgery for the treatment of lymphedema. Microsurgery 2006; 26(1):65-69

33 Yamamoto T, Matsuda N, Doi K, et al. The earliest finding of indocyanine green lymphography in asymptomatic limbs of lower extremity lymphedema patients secondary to cancer treatment: the modified dermal backflow stage and concept of subclinical lymphedema. Plast Reconstr Surg 2011;128(4):314e-321e

34 Mihara M, Hara H, Kawakami Y, et al. Multi-site lymphatic venous anastomosis using echography to detect suitable subcutaneous vein in severe lymphedema patients. J Plast Reconstr Aesthet Surg 2018;71(2):e1-e7

35 Akita S, Mitsukawa N, Kuriyama M, et al. Comparison of vascularized supraclavicular lymph node transfer and lymphaticovenular anastomosis for advanced stage lower extremity lymphedema. Ann Plast Surg 2015;74(5):573-579

36 Maegawa J, Hosono M, Tomoeda H, Tosaki A, Kobayashi S, Iwai T. Net effect of lymphaticovenous anastomosis on volume reduction of peripheral lymphoedema after complex decongestive physiotherapy. Eur J Vasc Endovasc Surg 2012;43(5): 602-608

37 Maegawa J, Mikami T, Yamamoto Y, Satake T, Kobayashi S. Types of lymphoscintigraphy and indications for lymphaticovenous anastomosis. Microsurgery 2010;30(6):437-442

38 Ito R, Wu CT, Lin MC, Cheng MH. Successful treatment of early-stage lower extremity lymphedema with side-to-end lymphovenous anastomosis with indocyanine green lymphography assisted. Microsurgery 2016;36(4):310-315 
39 Patel KM, Lin CY, Cheng MH. A prospective evaluation of lymphedema-specific quality-of-life outcomes following vascularized lymph node transfer. Ann Surg Oncol 2015;22(7): 2424-2430

40 Committee E; Executive Committee. The diagnosis and treatment of peripheral lymphedema: 2016 consensus document of the International Society of Lymphology. Lymphology 2016;49(4):170-184

41 Gloviczki P, Hollier LH, Nora FE, Kaye MP. The natural history of microsurgical lymphovenous anastomoses: an experimental study. J Vasc Surg 1986;4(2):148-156

42 Chen WF, Zhao H, Yamamoto T, Hara H, Ding J. Indocyanine Green Lymphographic Evidence of Surgical Efficacy Following Microsurgical and Supermicrosurgical Lymphedema Reconstructions. J Reconstr Microsurg 2016;32(9):688-698

43 Akita S, Ogata F, Manabe I, et al. Noninvasive screening test for detecting early stage lymphedema using follow-up computed tomography imaging after cancer treatment and results of treatment with lymphaticovenular anastomosis. Microsurgery 2017;37(8):910-916

44 Yamamoto Y, Sugihara T. Microsurgical lymphaticovenous implantation for the treatment of chronic lymphedema. Plast Reconstr Surg 1998;101(1):157-161

45 Chung JH, Baek SO, Park HJ, Lee BI, Park SH, Yoon ES. Efficacy and patient satisfaction regarding lymphovenous bypass with sleeve-in anastomosis for extremity lymphedema. Arch Plast Surg 2019;46(1):46-56

46 Yamamoto T, Narushima M, Kikuchi K, et al. Lambda-shaped anastomosis with intravascular stenting method for safe and effective lymphaticovenular anastomosis. Plast Reconstr Surg 2011;127(5):1987-1992

47 Yamamoto T, Yoshimatsu H, Narushima M, et al. Sequential anastomosis for lymphatic supermicrosurgery: multiple lymphaticovenular anastomoses on 1 venule. Ann Plast Surg 2014;73(1):46-49

48 Yamamoto T, Kikuchi K, Yoshimatsu H, Koshima I. Laddershaped lymphaticovenular anastomosis using multiple sideto-side lymphatic anastomoses for a leg lymphedema patient. Microsurgery 2014;34(5):404-408

49 Ayestaray B, Bekara F. $\pi$-shaped lymphaticovenular anastomosis: the venous flow sparing technique for the treatment of peripheral lymphedema. J Reconstr Microsurg 2014;30(8):551-560

50 Chen WF, Yamamoto T, Fisher M, Liao J, Carr J. The "Octopus" Lymphaticovenular Anastomosis: Evolving Beyond the Standard Supermicrosurgical Technique. J Reconstr Microsurg 2015;31(6):450-457

51 Yasunaga Y, Yanagisawa D, Ohata E, Matsuo K, Yuzuriha S. Bioelectrical Impedance Analysis of Water Reduction in LowerLimb Lymphedema by Lymphaticovenular Anastomosis. J Reconstr Microsurg 2019;35(4):306-314

52 Yamamoto T, Yoshimatsu H, Narushima M, et al. A modified side-to-end lymphaticovenular anastomosis. Microsurgery 2013;33(2):130-133

53 Koshima I, Nanba Y, Tsutsui T, Takahashi Y, Itoh S, Fujitsu M. Minimal invasive lymphaticovenular anastomosis under local anesthesia for leg lymphedema: is it effective for stage III and IV? Ann Plast Surg 2004;53(3):261-266

54 Yoshida S, Koshima I, Sasaki A, et al. Mechanical dilation using nylon monofilament aids multisite lymphaticovenous anastomosis through improving the quality of anastomosis. Ann Plast Surg 2019;82(2):201-206

55 Mihara M, Hayashi Y, Murai N, et al. Regional diagnosis of lymphoedema and selection of sites for lymphaticovenular anastomosis using elastography. Clin Radiol 2011;66(8): 715-719

56 Mihara M, Hara H, Narushima M, et al. Lower limb lymphedema treated with lymphatico-venous anastomosis based on pre- and intraoperative icg lymphography and non-contact vein visualization: A case report. Microsurgery 2012;32(3):227-230

57 Hayashi A, Hayashi N, Yoshimatsu H, Yamamoto T. Effective and efficient lymphaticovenular anastomosis using preoperative ultrasound detection technique of lymphatic vessels in lower extremity lymphedema. J Surg Oncol 2018;117(2):290-298

58 Hara H, Mihara M, Ohtsu H, Narushima M, Iida T, Koshima I. Indication of Lymphaticovenous Anastomosis for Lower Limb Primary Lymphedema. Plast Reconstr Surg 2015;136(4): 883-893

59 Chang DW, Suami H, Skoracki R. A prospective analysis of 100 consecutive lymphovenous bypass cases for treatment of extremity lymphedema. Plast Reconstr Surg 2013;132(5): 1305-1314

60 Demirtas Y, Ozturk N, Yapici O, Topalan M. Supermicrosurgical lymphaticovenular anastomosis and lymphaticovenous implantation for treatment of unilateral lower extremity lymphedema. Microsurgery 2009;29(8):609-618

61 Matsubara S, Sakuda H, Nakaema M, Kuniyoshi Y. Long-term results of microscopic lymphatic vessel-isolated vein anastomosis for secondary lymphedema of the lower extremities. Surg Today 2006;36(10):859-864

62 Koshima I, Nanba Y, Tsutsui T, Takahashi Y, Itoh S. Long-term follow-up after lymphaticovenular anastomosis for lymphedema in the leg. J Reconstr Microsurg 2003;19(4):209-215

63 Lee KT, Park JW, Mun GH. Serial two-year follow-up after lymphaticovenular anastomosis for the treatment of lymphedema. Microsurgery 2017;37(7):763-770

64 Mihara M, Hara H, Furniss D, et al. Lymphaticovenular anastomosis to prevent cellulitis associated with lymphoedema. $\mathrm{Br} \mathrm{J}$ Surg 2014;101(11):1391-1396

65 Huang GK, Hu RQ, Liu ZZ, Shen YL, Lan TD, Pan GP. Microlymphaticovenous anastomosis in the treatment of lower limb obstructive lymphedema: analysis of 91 cases. Plast Reconstr Surg 1985;76(5):671-685

66 Jacobson JH II, Suarez EL. Microvascular surgery. Dis Chest 1962;41:220-224

67 Gilbert A, O'Brien BM, Vorrath JW, Sykes PJ. Lymphaticovenous anastomosis by microvascular technique. Br J Plast Surg 1976;29(4):355-360

68 Yamada Y. Studies on lymphatic venous anastomosis in lymphedema. Nagoya J Med Sci 1969;32:1-21

69 Laine JB, Howard JM. Experimental lymphatico-venous anastomosis. Surg Forum 1963;14:111-112

70 O'Brien BM, Sykes P, Threlfall GN, Browning FS. Microlymphaticovenous anastomoses for obstructive lymphedema. Plast Reconstr Surg 1977;60(2):197-211

71 Koshima I, Inagawa K, Urushibara K, Moriguchi T. Supermicrosurgical lymphaticovenular anastomosis for the treatment of lymphedema in the upper extremities. J Reconstr Microsurg 2000;16(6):437-442

72 Mihara M, Hara H, Kikuchi K, et al. Scarless lymphatic venous anastomosis for latent and early-stage lymphoedema using indocyanine green lymphography and non-invasive instruments for visualising subcutaneous vein. J Plast Reconstr Aesthet Surg 2012;65(11):1551-1558

73 Campisi C, Bellini C, Campisi C, Accogli S, Bonioli E, Boccardo F. Microsurgery for lymphedema: clinical research and longterm results. Microsurgery 2010;30(4):256-260

74 Yamamoto T, Yamamoto N, Yamashita M, Furuya M, Hayashi A, Koshima I. Efferent lymphatic vessel anastomosis: supermicrosurgical efferent lymphatic vessel-to-venous anastomosis for the prophylactic treatment of subclinical lymphedema. Ann Plast Surg 2016;76(4):424-427

75 O’Brien BM, Mellow CG, Khazanchi RK, Dvir E, Kumar V, Pederson WC. Long-term results after microlymphaticovenous anastomoses for the treatment of obstructive lymphedema. Plast Reconstr Surg 1990;85(4):562-572 
76 Baumeister RG, Siuda S. Treatment of lymphedemas by microsurgical lymphatic grafting: what is proved? Plast Reconstr Surg 1990;85(1):64-74, discussion 75-76

77 Campisi C, Boccardo F. Microsurgical techniques for lymphedema treatment: derivative lymphatic-venous microsurgery. World J Surg 2004;28(6):609-613

78 Forte AJ, Huayllani MT, Boczar D, Ciudad P, Manrique O. Lipoaspiration and lymph node transfer for treatment of breast cancer-related lymphedema: a systematic review. Cureus 2019;11(11):e6096

79 Engel H, Lin CY, Huang JJ, Cheng MH. Outcomes of lymphedema microsurgery for breast cancer-related lymphedema with or without microvascular breast reconstruction. Ann Surg 2018;268(6):1076-1083

80 Pappalardo M, Patel K, Cheng MH. Vascularized lymph node transfer for treatment of extremity lymphedema: An overview of current controversies regarding donor sites, recipient sites and outcomes. J Surg Oncol 2018;117(7):1420-1431

81 Demirtas Y, Ozturk N, Yapici O, Topalan M. Comparison of primary and secondary lower-extremity lymphedema treated with supermicrosurgical lymphaticovenous anastomosis and lymphaticovenous implantation. J Reconstr Microsurg 2010;26(2):137-143

82 Nagase T, Gonda K, Inoue $\mathrm{K}$, et al. Treatment of lymphedema with lymphaticovenular anastomoses. Int J Clin Oncol 2005;10(5):304-310

83 Seki Y, Yamamoto T, Yoshimatsu H, et al. The Superior-Edge-ofthe-Knee Incision Method in Lymphaticovenular Anastomosis for Lower Extremity Lymphedema. Plast Reconstr Surg 2015;136(5):665e-675e

84 Suami H, Chang DW, Yamada K, Kimata Y. Use of indocyanine green fluorescent lymphography for evaluating dynamic lymphatic status. Plast Reconstr Surg 2011;127(3):74e-76e

85 Yamamoto T, Koshima I, Yoshimatsu H, Narushima M, Miahara M, Iida T. Simultaneous multi-site lymphaticovenular anastomoses for primary lower extremity and genital lymphoedema complicated with severe lymphorrhea. J Plast Reconstr Aesthet Surg 2011;64(6):812-815

86 Narushima M, Mihara M, Yamamoto Y, Iida T, Koshima I, Mundinger GS. The intravascular stenting method for treatment of extremity lymphedema with multiconfiguration lymphaticovenous anastomoses. Plast Reconstr Surg 2010;125(3):935-943

87 Hara H, Mihara M, Seki Y, Todokoro T, Iida T, Koshima I. Comparison of indocyanine green lymphographic findings with the conditions of collecting lymphatic vessels of limbs in patients with lymphedema. Plast Reconstr Surg 2013;132(6):1612-1618
88 Chen WF. How to Get Started Performing Supermicrosurgical Lymphaticovenular Anastomosis to Treat Lymphedema. Ann Plast Surg 2018;81(6S) (Suppl 1) :S15-S20

89 Yamamoto T, Yoshimatsu H, Yamamoto N, et al. Modified lambda-shaped lymphaticovenular anastomosis with supermicrosurgical lymphoplasty technique for a cancer-related lymphedema patient. Microsurgery 2014;34(4):308-310

90 Yamamoto T, Yoshimatsu H, Yamamoto N, Narushima M, Iida T, Koshima I. Side-to-end Lymphaticovenular anastomosis through temporary lymphatic expansion. PLoS One 2013;8(3):e59523

91 Yamamoto T, Yoshimatsu H, Narushima M, Yamamoto N, Koshima I. Split intravascular stents for side-to-end lymphaticovenular anastomosis. Ann Plast Surg 2013;71(5):538-540

92 Aljindan FK, Lin CY, Cheng MH. Comparison of outcomes between Side-to-End and End-to-End Lymphovenous Anastomoses for Early-Grade Extremity Lymphedema. Plast Reconstr Surg 2019;144(2):486-496

93 Nuri T, Ueda K, Yamada A, Hara M. Preparatory intravascular stenting technique: an easier method of supermicrosurgical lymphaticovenular anastomosis. Ann Plast Surg 2013; 71(5):541-543

94 Ciudad P, Sabbagh MD, Agko M, et al. Surgical management of lower extremity lymphedema: a comprehensive review. Indian J Plast Surg 2019;52(1):81-92

95 Scaglioni MF, Uyulmaz S, Arvanitakis M Lineaweaver WC, Zhang F. Intraoperatively detected but previously indocyanine green-negative lymphatic vessels may have misprized potentials and should not be neglected in lymphaticovenous bypass surgery. Annals of plastic surgery 2019;83(1):63-72

96 Yoshimatsu H, Yamamoto T, Narushima M Iida T Koshima I. The guide wire method: a new technique for easier side-toend lymphaticovenular anastomosis. Annals of plastic surgery 2014;73(2):231-233

97 Akita S, Mitsukawa N, Kuriyama M, et al. Suitable therapy options for sub-clinical and early-stage lymphoedema patients. J PLAST RECONSTR AES 2014;67(4):520-525

98 Akita S, Mitsukawa N, Kuriyama M, et al. External valvuloplasty for subcutaneous small veins to prevent venous reflux in lymphaticovenular anastomosis for lower extremity lymphedema. Plast Reconstr Surg 2013;132(4):1008-1014

99 Maegawa J, Yabuki Y, Tomoeda H Hosono M Yasumura K. Outcomes of lymphaticovenous side-to-end anastomosis in peripheral lymphedema. J Vasc Surg 2012;55(3):753-760

100 Motegi S, Tamura A, Okada E Nagai Y Ishikawa O. Successful treatment with lymphaticovenular anastomosis for secondary skin lesions of chronic lymphedema. Dermatology 2007;215(2):147-151 